Volume 3

\title{
The Association Between Doppler Measures of Cardiac Function and Outcomes in Patients With Left Ventricular Ejection Fraction $\leq 40 \%$ Undergoing Noncardiovascular Surgeries
}

\author{
Yang Shi \\ Rachel Pedersen \\ Matthew Rappelt \\ Robyn Shearer \\ Nasir Z. Sulemanjee \\ Dianne L. Zwicke \\ T. Edward Hastings \\ Omar M. Cheema \\ Vinay Thohan
}

Follow this and additional works at: https://aah.org/jpcrr

Part of the Cardiology Commons, and the Cardiovascular Diseases Commons

\section{Recommended Citation}

Shi Y, Pedersen R, Rappelt M, Shearer R, Sulemanjee NZ, Zwicke DL, Hastings TE, Cheema OM, Thohan V. The association between Doppler measures of cardiac function and outcomes in patients with left ventricular ejection fraction $\leq 40 \%$ undergoing noncardiovascular surgeries. J Patient Cent Res Rev. 2016;3:236-7.

Published quarterly by Midwest-based health system Advocate Aurora Health and indexed in PubMed Central, the Journal of Patient-Centered Research and Reviews (JPCRR) is an open access, peer-reviewed medical journal focused on disseminating scholarly works devoted to improving patient-centered care practices, health outcomes, and the patient experience. 
$(\mathrm{P}=0.2)$, whereas it continued to show benefit in the group with LVEF of $40 \%-49 \%(\mathrm{P}=0.001)$.

Conclusion: Clinical predictors of mortality in patients with transient LV systolic dysfunction may help further risk-stratify this cohort of patients. It appears that patients with LVEF of $40 \%-49 \%$ continue to derive benefit from ICD therapy.

\section{FIRST PLACE ORAL PRESENTATION}

See page 245 for citation.

\section{SECOND PLACE ORAL PRESENTATION Path to Resistance: Risk Factors Associated With Carbapenem-Resistant Pseudomonas aeruginosa}

\author{
Kushal Patel, Jessica J.F. Kram, Dennis J. Baumgardner
}

Department of Internal Medicine, Aurora Sinai Medical Center; Department of Family Medicine, Aurora UW Medical Group; Center for Urban Population Health

Background: An estimated 51,000 health care-associated Pseudomonas aeruginosa infections occur in the United States annually. More than $13 \%$ are secondary to non-carbapenem multidrug-resistant strains, which result in 400 yearly deaths. Traditional risk factors for resistance include ICU stay, mechanical ventilation, previous hospitalization and major comorbidities. As microbes evolve, risk factors also may evolve.

Purpose: To determine if traditional and/or new risk factors for $P$. aeruginosa resistance are valid and predictive of infection with carbapenem-resistant $P$. aeruginosa.

Methods: We retrospectively studied inpatients and outpatients $\geq 18$ years old who presented to an Aurora Health Care facility with a positive $P$. aeruginosa culture during 2014. Cultures were obtained from the ACL Laboratories database, and patient medical records were reviewed in Epic. Chi-squared test with Yates correction and two-sample t-tests were performed on categorical and continuous variables, respectively. Binary regression was used for multivariable modeling. Significance was associated with $\mathrm{P}<0.05$.

Results: Study population $(\mathrm{N}=1,763)$ characteristics were: mean age 68.0 , body mass index $30.4 \mathrm{~kg} / \mathrm{m}^{2}, 51.2 \%$ female sex, and $89.3 \%$ white race. Resistance to imipenem or meropenem $(14.0 \%)$ on univariable analysis was associated with younger age ( 66.0 vs 68.3 years, $\mathrm{P}=0.027)$, hospitalized patients $(19.7 \%$ vs $8.6 \%, \mathrm{P}<0.0001)$, male sex $(16.0 \%$ vs $12.0 \%, \mathrm{P}=0.017)$, nonwhite race $(23.5 \%$ vs $12.3 \%, \mathrm{P}<0.0001)$, respiratory culture $(30.9 \%$ vs $12.1 \%, \mathrm{P}<0.0001)$, history of pulmonary disease $(19.4 \%$ vs $12.9 \%, \mathrm{P}=0.005)$, history of congestive heart failure ( $18.6 \%$ vs $13.0 \%, \mathrm{P}=0.016)$, history of multidrug resistance $(33.3 \%$ vs $13.6 \%, \mathrm{P}=0.003)$ and recent surgery $(17.8 \%$ vs $12.2 \%, \mathrm{P}=0.002)$, as well as transfer from institution, Foley catheter, vasopressor treatment, central/PIC lines, mechanical ventilation, ICU admission, and bedridden status (all $\mathrm{P}<0.0001$ ). In multivariable modeling, nonwhite race, respiratory culture, recent transfer, vasopressor use and central/PIC lines were significant. Only $0.57 \%$ of strains were resistant to the six traditional non-carbapenem drugs and both carbapenems.

Conclusion: Demographic and traditional risk factors, as well as respiratory cultures, were predictive of carbapenem resistance. Such information may guide initial antibiotic treatment of $P$. aeruginosa. Fortunately, less than $1 \%$ of strains were resistant to all drugs tested. Further studies looking at change in outcome while incorporating these risk factors in determination of empiric coverage for patients should be performed.

\section{THIRD PLACE ORAL PRESENTATION}

See page 245 for citation.

\section{FIRST PLACE POSTER}

See page 245 for citation.

\section{SECOND PLACE POSTER (tie)}

The Association Between Doppler Measures of Cardiac Function and Outcomes in Patients With Left Ventricular Ejection Fraction $\leq \mathbf{4 0} \%$ Undergoing Noncardiovascular Surgeries

Yang Shi, Rachel Pedersen, Matthew Rappelt, Robyn Shearer, Nasir Z. Sulemanjee, Dianne L. Zwicke, T. Edward Hastings, Omar M. Cheema, Vinay Thohan

Sheikh Khalifa bin Hamad Al Thani Center for Integrative Research on Cardiovascular Aging, Aurora Research Institute; Transplant Administration, Aurora Health Care; Aurora Cardiovascular Services, Aurora Health Care

Background: Preoperative risk assessments of individuals who undergo major noncardiac surgery have focused on ischemic heart disease. Information on how to assess the noncardiac surgical risks for patients with depressed cardiac function, as seen in heart failure, is sparse. Echocardiography is routinely performed in patients with depressed cardiac function and is an accepted standard cardiac assessment. Transthoracic echocardiography (TTE) provides strong independent prognostic implications in a wide range of cardiovascular conditions.

Purpose: To identify the echocardiographic parameters associated with outcomes among patients undergoing major noncardiac surgery.

Methods: A retrospective single-institution investigation identified 1,770 patients who underwent one or more major noncardiac procedures from Jan. 1, 2011, to June 30, 2014, and had at least one TTE performed within 90 days before surgery. Patients were stratified by presurgery left ventricular ejection fraction (LVEF) into LVEF $\leq 40 \%$ and LVEF $>40 \%$ groups. The cohort was followed through June 12, 2015, with the outcome focused on all-cause mortality. Continuous and categorical variables were compared by Student's t-test and chi-squared test, respectively. Kaplan-Meier method was used to calculate mortality estimates postsurgery. Cox proportional hazards model was used for univariate and multivariable models. 
Results: In patients with LVEF $>40 \%$, the 1-, 6- and 12-month mortality rates were $3.8 \%, 9.0 \%$ and $12.1 \%$, respectively. In patients with LVEF $\leq 40 \%, 1-, 6$ - and 12-month mortality was $9.5 \%, 18.4 \%$ and $25.2 \%$, significantly greater than patients with $\mathrm{LVEF}>40 \%$ at all time points $(\mathrm{P}<0.01)$. Univariate analysis of patients with LVEF $\leq 40 \%$ found the following echocardiographic parameters to be significant predictors of 6-month mortality: right atrial pressure, pulmonary artery systolic pressure, LVEF $<25 \%$, mitral A-wave velocity, mitral E-wave deceleration time, and left ventricular posterior wall diastolic thickness. Multivariate analysis identified mitral A-point velocity (hazard ratio $[\mathrm{HR}]$ : $0.98, \mathrm{P}=0.02$ ), LVEF $<$ 25\% (HR: 3.48, $\mathrm{P}<0.01$ ), glomerular filtration rate (HR: 0.71 at 10 -unit increments, $\mathrm{P}<0.01)$ and colectomy $(\mathrm{HR}=5.47, \mathrm{P}<0.01)$ as significant predictors of 6-month mortality.

Conclusion: Preoperative LVEF $<25 \%$, lower mitral A velocity, colectomy, and lower glomerular filtration rate are associated with 6-month mortality postsurgery. Close preoperative cardiac assessment of patients with decreased LVEF prior to noncardiac surgery may prove beneficial in improving long-term outcomes.

\section{SECOND PLACE POSTER (tie)}

See page 245 for citation.

\section{THIRD PLACE POSTER}

See page 245 for citation.

\section{SELECT ABSTRACTS}

Association Between Pregnancy Intention and Maternal Characteristics, Outcomes, and Cost of Care: A Pilot Study

Kristy M. Kelel, Kiley B. Vander Wyst, Danielle M. Greer, Danish Siddiqui

Department of Obstetrics and Gynecology, Aurora Sinai Medical Center; Center for Urban Population Health; Department of Obstetrics and Gynecology, Aurora UW Medical Group

Background: An estimated 51\% of pregnancies in the United States are unintended. In Wisconsin, unplanned pregnancies account for $40 \%$ of all pregnancies and cost $\$ 148$ million in public funds. Unintended pregnancy, which creates increased hardship for mothers and threatened well-being of infants, has been recognized as an important health, social and economic problem.

Purpose: To determine the pregnancy intentions of postpartum women and the maternal characteristics, outcomes and costs of care associated with unintended pregnancies at a large urban hospital in Milwaukee, Wisconsin.

Methods: Postpartum women were surveyed prior to discharge. The 20-item survey included whether or not the woman had been trying to get pregnant and how she felt about the timing of her pregnancy. Electronic medical records were reviewed to determine maternal and neonatal outcomes, including antenatal, perinatal, postpartum comorbidities and complications. To determine the most important factors influencing the binary and multicategory responses of pregnancy intention, logistic and multinomial regression models were developed using stepwise variable selection procedures.

Results: A total of 338 women were asked to participate, resulting in 243 completed surveys ( 95 exclusions: 8 declines, 29 language barriers, 46 lost to follow-up, 12 other). Overall, 63\% (142/227) of pregnancies occurred when "not trying." Logistic and multinomial regression revealed anemia $(\mathrm{P}=0.004-0.007)$, anxiety $(\mathrm{P}=0.048)$ and income level $(\mathrm{P}=0.002-0.045)$ as the most significant predictors of unintended pregnancy. The odds of unintended pregnancy for women at the lowest two income levels were 12.05 (odds ratio: 2.82-51.39) and 3.83 (odds ratio: 1.314-11.142) times greater than those for women at the highest income level. Significant univariate associations existed between unintended pregnancy and age $(\mathrm{P}<0.001)$, race $(\mathrm{P}=0.025)$ and insurance $(\mathrm{P}=0.003)$.

Conclusion: The unintended pregnancy rate of our study population was greater than state and national levels. Maternal characteristics of income, anemia and anxiety were the most significant predictors of pregnancy intention, but unintended pregnancy also was highly associated with younger age, African-American race and Medicaid insurance. Unintended pregnancy effects included: fewer prenatal care visits, increased prevalence of intrauterine growth restriction and decreased likelihood of breastfeeding. While the relative use of contraception was significantly greater, the absolute use among women who had an unintended pregnancy is of great clinical concern.

\section{Models for Predicting Incident Delirium in Hospitalized Older Adults: A Systematic Review}

Sundeep Kalimisetty, Wajih Askar, Brenda Fay, Ariba Khan

Department of Geriatrics, Aurora Sinai Medical Center; Aurora Libraries, Aurora Health Care; Department of Geriatrics, Aurora UW Medical Group

Background: Delirium is common in hospitalized older adults, and $40 \%$ of cases may be preventable. Hospital Elder Life Program is an evidence-based program to reduce incidence of delirium. It has been successfully implemented in one hospital and will be implemented in four other hospitals. Identification of patients at highest risk of developing delirium using the electronic health record (EHR) may be an effective targeted strategy to reduce the incidence of delirium.

Purpose: To systematically review and summarize the medical literature regarding risk prediction models for delirium in older inpatients.

Methods: A medical librarian customized and conducted the search strategy for all published medical articles on delirium prediction models. Electronic databases sourced included Ovid MEDLINE, CINAHL, Cochrane Database of Systematic Reviews, EMBASE and PsycINFO. Controlled vocabulary terms specific to database as well as relevant keywords were 